\title{
An Exploration of the Scientific Writing Experience of Nonnative English-Speaking Doctoral Supervisors and Students Using a Phenomenographic Approach
}

\author{
Elizabeth Dean, ${ }^{1,2}$ Lena Nordgren, ${ }^{3,4}$ and Anne Söderlund ${ }^{5}$ \\ ${ }^{1}$ Department of Physical Therapy, Faculty of Medicine, University of British Columbia, Vancouver, BC, Canada V6T $1 Z 3$ \\ ${ }^{2}$ School of Health, Care and Social Welfare, Mälardalen University, Sweden \\ ${ }^{3}$ Centre for Clinical Research in Sörmland, Sörmland County Council, 63188 Eskilstuna, Sweden \\ ${ }^{4}$ Department of Public Health and Caring Sciences, Uppsala University, 75122 Uppsala, Sweden \\ ${ }^{5}$ Department of Physiotherapy, School of Health, Care and Social Welfare, Mälardalen University, 72123 Västerås, Sweden
}

Correspondence should be addressed to Elizabeth Dean; elizabeth.dean@ubc.ca

Received 26 September 2015; Accepted 1 December 2015

Academic Editor: Julie Redfern

Copyright (C) 2015 Elizabeth Dean et al. This is an open access article distributed under the Creative Commons Attribution License, which permits unrestricted use, distribution, and reproduction in any medium, provided the original work is properly cited.

\begin{abstract}
Nonnative English-speaking scholars and trainees are increasingly submitting their work to English journals. The study's aim was to describe their experiences regarding scientific writing in English using a qualitative phenomenographic approach. Two focus groups (5 doctoral supervisors and 13 students) were conducted. Participants were nonnative English-speakers in a Swedish health sciences faculty. Group discussion focused on scientific writing in English, specifically, rewards, challenges, facilitators, and barriers. Participants were asked about their needs for related educational supports. Inductive phenomenographic analysis included extraction of referential (phenomenon as a whole) and structural (phenomenon parts) aspects of the transcription data. Doctoral supervisors and students viewed English scientific writing as challenging but worthwhile. Both groups viewed mastering English scientific writing as necessary but each struggles with the process differently. Supervisors viewed it as a long-term professional responsibility (generating knowledge, networking, and promotion eligibility). Alternatively, doctoral students viewed its importance in the short term (learning publication skills). Both groups acknowledged they would benefit from personalized feedback on writing style/format, but in distinct ways. Nonnative English-speaking doctoral supervisors and students in Sweden may benefit from on-going writing educational supports. Editors/reviewers need to increase awareness of the challenges of international contributors and maximize the formative constructiveness of their reviews.
\end{abstract}

\section{Introduction}

Research findings are only as good as the quality of the scientific writing to describe them. Nonnative English-speaking scholars and their trainees are publishing increasingly in mainstream English language peer-reviewed academic journals. They are less content to publish in their native language journals, if they exist, or in the language of the country where they work or study. They opt to submit their work to English journals that are indexed and generally better known, disseminated, and accessed. These journals often with impact factors are also more prestigious when scholars and their trainees are being considered for promotion or admission to graduate or postdoctoral fellowship programs and awards. The demand for excellence in scientific writing affects all writers, but this may be a particular burden for nonnative English-speaking scholars and trainees.

Editorial staff members of academic journals have attempted to facilitate the publication success of nonnative English-speaking contributors based on their perceptions of their needs. Editorials and articles on scientific writing have emerged aimed at these contributors [1-5]. These and articles on scientific writing generally tend to provide basic guidelines, for example, reiteration and elaboration of instructions for authors on format and style, and the conventional IMRAD format (i.e., introduction, methods, results, and discussion) 
$[6,7]$. Conceivably, the needs of nonnative English speakers are distinct [8-13]. Whether such articles address the specific needs of nonnative English-speaking authors is unclear. And whether these contributors would benefit from other types of supports also warrants elucidation.

Our primary research question was: do nonnative English-speaking doctoral supervisors and students have unique experiences with respect to English scientific writing? If so, can knowledge of their experiences inform educational supports to maximize their publication success and writing experiences?

Facilitating the scientific writing process for scholars and their trainees is no more important than at present. Global health trends and escalating health care costs have increased the demand for expedient and timely knowledge generation and translation across health professions. The transfer and dissemination of this growing knowledge base depend exclusively on the quality of the scientific written communication in English.

\section{Method}

2.1. Study Design and Approach. A qualitative method based on a phenomenographic approach was used $[14,15]$. The study was approved by the institutional ethical review board. Consent was provided by participants' agreeing to attend a focus group (supervisors or students).

Phenomenography aims to describe qualitatively the various ways in which people conceive phenomena in their unique worlds [16]. The investigators aim to categorize conceptions of a phenomenon of interest as accurately as possible and refrain from interjecting their preconceived ideas. A phenomenon can be understood qualitatively in terms of its referential and structural aspects [16]. Referential aspects are the attributes of the phenomenon conceived as a whole. Structural aspects are the parts of the whole and describe how the parts are related. The phenomenon of interest in our study was the lived experience of scientific writing in English by nonnative English-speaking doctoral supervisors and students.

In phenomenography, investigators allow the data to emerge so that rich description of a phenomenon is accurately captured. The interview/discussion questions are designed to elicit the participants' experiences, feelings, beliefs, and convictions about a phenomenon. The research questions and discussion questions are often distinct.

\subsection{Setting and Participants and Their Recruitment. The} setting was a school of health, care and social welfare at a medium-sized university in Sweden. To obtain a purposive sample, the study was publicized a week beforehand. In the publicity notice and through word-of-mouth, doctoral supervisors and students were invited to participate in separate focus groups. In the study announcement, potential participants were informed that the focus groups would last 75 minutes. They were also invited to attend an interactive seminar on English scientific writing given by an investigator. This seminar followed each focus group. Participants were not obliged to attend a focus group to attend the seminar.
This opportunity was particularly important for the graduate students to avoid the perception of coercion to participate. The focus groups and the seminar were conducted in English.

2.3. Data Gathering. Data gathering methods included audiotaping and memoing or field notes. Audiotaping of the focus groups was conducted with two electronic devices to minimize the risk of inaudible or lost data. Two investigators had access to the audiotapes, hence knowledge of participants' identities prior to coding.

The same experienced investigator served as the focus groups' leader. First, she described the aim of the focus group and reinforced that individuals had provided consent to participate by attending and that their responses would be masked.

The focus groups had the same structure. They began with a warm-up question about the meaning of scientific writing. Then, the discussion focused on five topics related to English scientific writing, that is, benefits, challenges, facilitators, barriers, and, finally, support needs to improve writing proficiency. At the end, participants completed a questionnaire about their language proficiencies, experiences in scientific writing and publishing, and support needs, if any, for improving their writing. The two focus groups were two days apart.

Memoing consists of the investigators' recording notes about what they hear, see, experience, and think over the course of collecting and reflecting on the process. These observations and reflections are used in the explication of the findings. The three investigators were present for both focus groups and took notes.

2.4. Explication of the Findings. In phenomenography, data are explicated rather than analyzed. The five steps include bracketing; delineating meaning units or referential aspects; clustering structural units and defining a focus for each referential aspect; summarizing each interview/discussion and validating it and, where necessary, modifying it (results); and extracting general and unique themes (discussion).

Bracketing refers to containing the investigators' views and preconceptions so they do not influence the interview/discussion processor explication of the findings.

Delineating meaning units and foci refers to extracting statements that illuminate the researched phenomenon. Although investigators are required to make judgments while consciously bracketing their own presuppositions, they avoid extraneous judgments about the data and their explication. Two investigators listened repeatedly to the audio recordings to familiarize themselves with the participants' words and develop a holistic sense. The recordings were transcribed and read and reread multiple times to similarly develop a holistic sense. Significant statements related to the study aim were marked. The referential aspects of the phenomenon of scientific writing in English were identified according to similarities and differences for the two groups. Then, the focus of each referential aspect of the phenomenon was identified and its structural aspects, that is, what each group's participants primarily focused on. To strengthen the study's credibility, the investigators discussed the referential and 
TABLE 1: Background of doctoral supervisors $(n=5)$ and students $(n=13)$ with respect to primary language, publishing experiences, and related perceived educational needs (frequency).

(a)

\begin{tabular}{lc}
\hline \multicolumn{2}{c}{ Doctoral supervisors $(n=5)$} \\
Attribute/descriptor & Frequency \\
\hline Primary language Swedish & 4 \\
\hline English as a second or third language & 5 \\
\hline Publishing experience (y) & $\begin{array}{c}4 \text { to } 15 \\
\text { (out of } 4 \text { responses) }\end{array}$ \\
\hline Peer-reviewed publications & $7-65$ \\
\hline Formal scientific writing & None (4) \\
instruction/course & 20 wk course, $10 \mathrm{~h} / \mathrm{wk}(1)$ \\
\hline
\end{tabular}

(b)

\begin{tabular}{lc}
\hline \multicolumn{2}{c}{ Doctoral students $(n=13)$} \\
Attribute/descriptor & Frequency \\
\hline Primary language Swedish & 10 \\
\hline English as a second or third language & 13 \\
\hline Publishing experience (y) & No experience $(8)$ \\
& 0.5 to $4(5)$ \\
\hline Peer-reviewed publications & None $(9)$ \\
& 1 or 2 (4) \\
\hline Formal scientific writing & None (8) \\
instruction/course & 2 wk course (4) \\
& One day in research \\
& education (1) \\
\hline
\end{tabular}

structural aspects as they evolved and their interpretations until agreement was reached.

\section{Results}

3.1. Participants. Participants included a purposive sample of 5 doctoral supervisors and 13 students. The details of their publication experiences and instruction in scientific writing appear in Table 1.

3.2. Focus Groups. In the transcripts of the supervisor group, the two investigators identified 80 to 85 significant statements in a total of 5700 to 5800 words. In the transcripts of the doctoral students, the two investigators identified 120 to 125 significant statements in a total of 4100 to 4200 words.

3.3. Doctoral Supervisors' Conceptions. The analysis revealed four qualitatively distinct referential aspects that describe the doctoral supervisors' conceptions of scientific writing in English: a challenging task, an academic responsibility, a "disadvantageous" struggle, and need to master the written English language (Table 2). Scientific writing was described as being concise, structured, and clear; writing that reflects a topic's essence. Supervisors acknowledged that writing traditions for quantitative and qualitative research are distinct. They also acknowledged journal differences in style and formatting.
First, with respect to scientific writing in English being challenging, the focus that emerged was the writing process. Supervisors described the demands of writing including language demands and time constraints. Language demands included the fact that direct translation to English is limited as it fails to capture the essence of the intended meaning. Supervisors concurred that ways of thinking, knowing, and conceptualizing ideas and framing arguments are distinct across languages. They acknowledged that writing in English is "hard" work and more time-consuming than in their native languages. A shared experience was the conflict between teaching and administrative responsibilities and research and writing time.

Second, supervisors acknowledged that writing in English is an academic responsibility. English being the universal language of science necessitates supervisors having a high level of proficiency in order to have their work accepted for publication. It was viewed as an "unspoken expectation."

Third, the conception that English scientific writing is a "disadvantageous" struggle was a sentiment shared by supervisors. Publishing in their native languages was not viewed as an option given journals in their fields often do not exist and, if they do, their scientific quality, circulation, and distribution are limited. Examples of the "disadvantageous" struggle included experiences of having reviewers' ridicule their writing. Also, they reported being required to overly justify their contexts compared with mainstream native English-speaking peers. They attributed these experiences to editors and reviewers having little awareness of and sensitivity to the challenges of nonnative English-speaking contributors in writing in English for publication. Supervisors expressed hesitancy to share English language challenges with their colleagues.

Lastly, the need for supervisors to master written English focused on the need to facilitate a culture of English communication in their settings. Despite having written in English for many years, supervisors still struggle with English grammar, composition, and punctuation. More salient was the struggle to find appropriate English words to translate ideas into English that have been conceptualized in their own languages. They concurred that a facilitating English-immersion environment would improve proficiency in their written English, for example, more teaching opportunities in English, seminars in English, and having native-speaking English language support people within their departments and familiar with their content. Supervisors' favored resources and supports for improving English proficiency appear in Table 3.

3.4. Doctoral Students' Conceptions. Four referential aspects emerged for the doctoral students: a struggle to master scientific writing in English, need to focus on the goal, need for writing guidance and assistance, and a challenging on-going learning process (Table 4). Scientific writing was described as a means of communication in the scientific community. It was associated with the structured IMRAD format. Students viewed scientific writing as a process informed by objective evidence and ethical considerations.

First, with respect to the struggle of mastering English scientific writing, concerns included the time needed to write in English, no direct translation, and limited opportunities to 


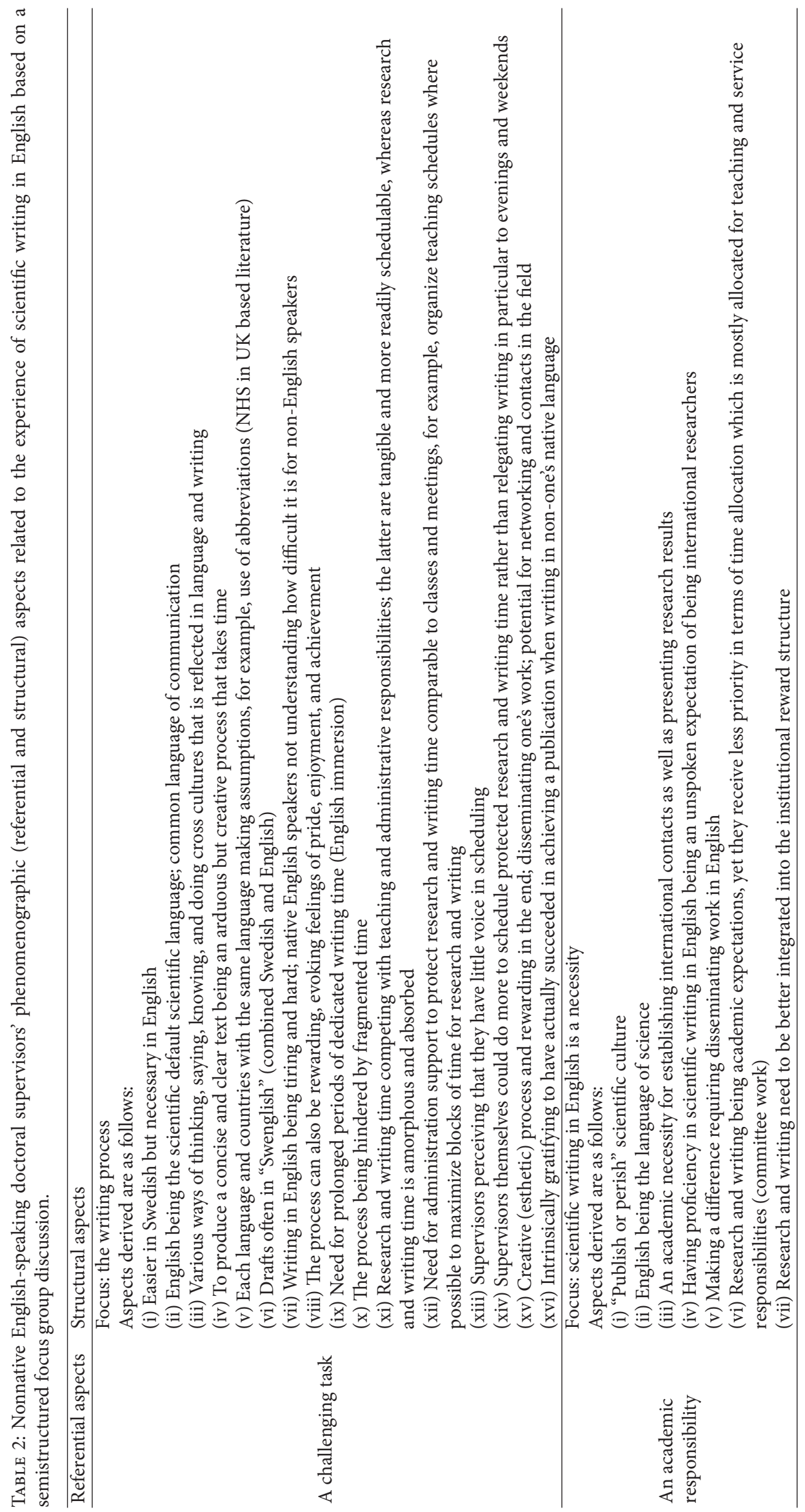




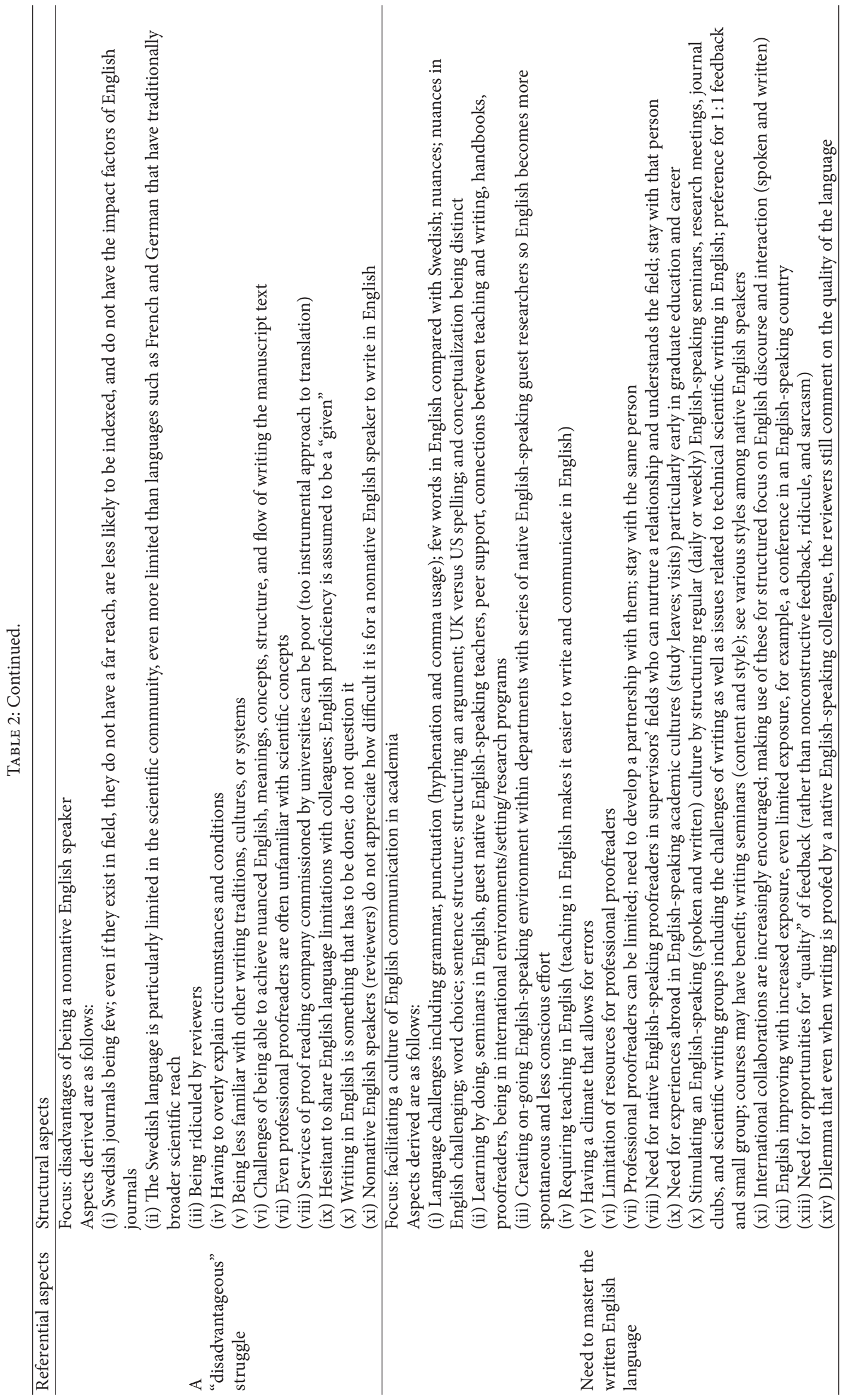


TABLE 3: Desired supports and resources to maximize English scientific writing proficiency articulated by nonnative English-speaking doctoral supervisors and students.

(i) Recognition of administration and department heads of research and writing as priorities needing dedicated time
(ii) Need to cluster teaching as much as possible to allow blocks of time for research and writing
(iii) Creating an English communication culture (experiences abroad and visiting native English-speaking visiting guest
professors to departments)
(iv) Hands-on working groups
(v) More opportunities to speak and teach in English
(vi) Access to a departmental native English-speaking resource person
(vii) Access to proofreaders but who are knowledgeable about the content and language of the discipline
supervisors (viii) Importance of a sustained relationship with a proofreader
(ix) Translator services but need personnel to be familiar with discipline and context
(x) Constructive criticism and correction versus destructive, negative, and insulting comments
(xi) Need for more handbooks and hands-on resources targeting the needs of nonnative English-speaking scholars
(xii) More opportunities to share writing issues with colleagues in field
(i) Even brief times in English-speaking contexts (immersion) need to be encouraged, but exposure to English needs to be
sustained
(ii) Ready access to a native English speaker with knowledge of the student's field
(iii) Devoting time to identify and read exemplary articles in English for style (not only content)
(iv) More opportunities to present in English
(v) An atmosphere where it is acceptable to make mistakes to foster constructive feedback and minimize fear of destructive
criticism
(vi) Discussion of writing strategies as well as English grammar and composition
(vii) Personalized concrete feedback so students learn how to edit their work by understanding what is wrong and why
(viii) Need for more handbooks and hands-on resources targeting the needs of nonnative English-speaking scholars
(ix) More opportunities to share writing issues with peers in field
Doctoral
students

practice English. However, they acknowledged that moving between languages afforded them new perspectives and ideas. Further, they described the limitations of tools such as Google translator even though many used it. Despite limited publication experience, students expressed concern that reviewers would criticize their English.

Second, students focused on the short-term goal of publication. They viewed publication as a means of disseminating work and networking internationally. They expressed mixed feelings about proofreaders. They described their limitations, for example, lack of in-depth content knowledge. Also, they expressed hesitation to override a proofreader's suggestion believing the proofreader is more knowledgeable.

Third, regarding writing guidance and assistance, the students articulated that they needed supportive strategies to maximize their writing and publication success. Supports they favored were seminars and teaching in English; being required to speak English; individual feedback; and guidance in developing writing strategies. Although students were aware of self-help writing guides, they mostly used representative published articles as examples of proficient English scientific writing.

Lastly, students experienced scientific writing as an ongoing learning process. They were concerned about developing their personal writing styles and overcoming barriers such as inexperience and lack of confidence. To improve their English writing, they discussed the importance of reading more articles to learn from their styles as well as contents. English immersion opportunities were considered essential. They were however reluctant to share their writing challenges with peers. Students' favored resources and supports for improving English proficiency appear in Table 3.

\section{Discussion and Conclusion}

In terms of final explication of the findings, this discussion focuses on similarities and differences between the doctoral supervisors and students regarding their experiences of English scientific writing that emerged in the referential aspects and their foci and structural aspects. Secondarily, we identify similarities and differences identified by the two groups regarding writing supports.

4.1. Group Findings. Quotations are identified as supervisor (SUP) or student (STUD), by participant number (P- $x$ ) and transcript page number $(x)$. To further mask participant identity, there is no reference to each participant's sex. Some words and phrases have been selectively added in the participants' quotations, shown in parentheses, to maximize their clarity or further ensure participant anonymity.

Regarding similarities, doctoral supervisors and students referred to the writing process in English as hard work and time-consuming (supervisor referential aspects, a challenging task, and need to master written English language), student referential aspects, a struggle to master English scientific writing, and a challenging on-going learning process. Supervisors' primary concern was insufficient time. Supervisor 1, for example, said "... No, not just (having) time, but (having) coherent time" (Sup-P1, page 11). Students were largely concerned about the process. For example, Student 12 stressed that “...it's practicing of your English, as well as trying to look at it from different perspectives" (Stud-P12, page 3). Brevity and flow were another challenge. Student 2 said "I have to cut it, to take away part that you cannot explain in English, so you just explain the other part" (Stud-P2, page 6). And, Student 9 


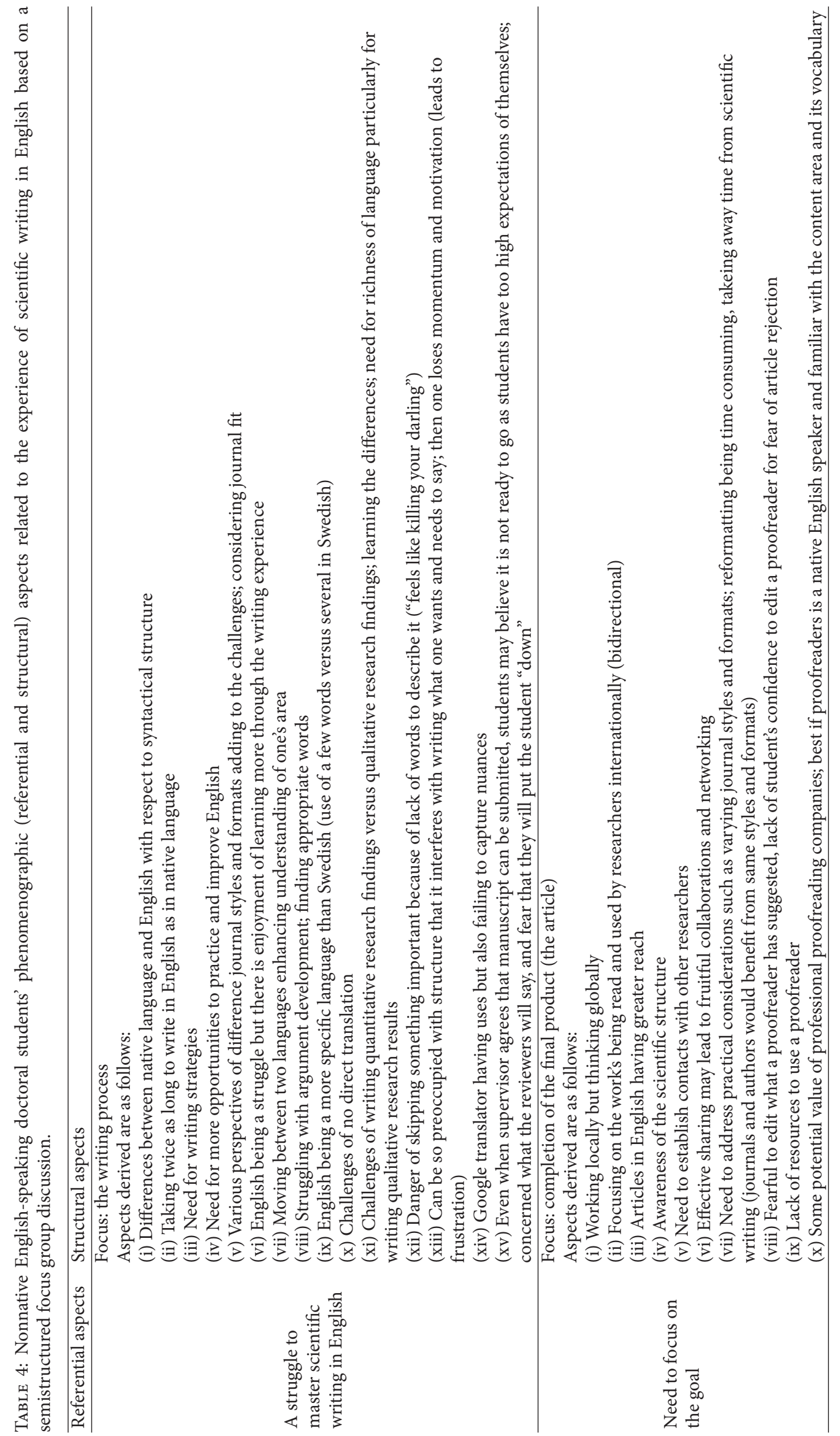




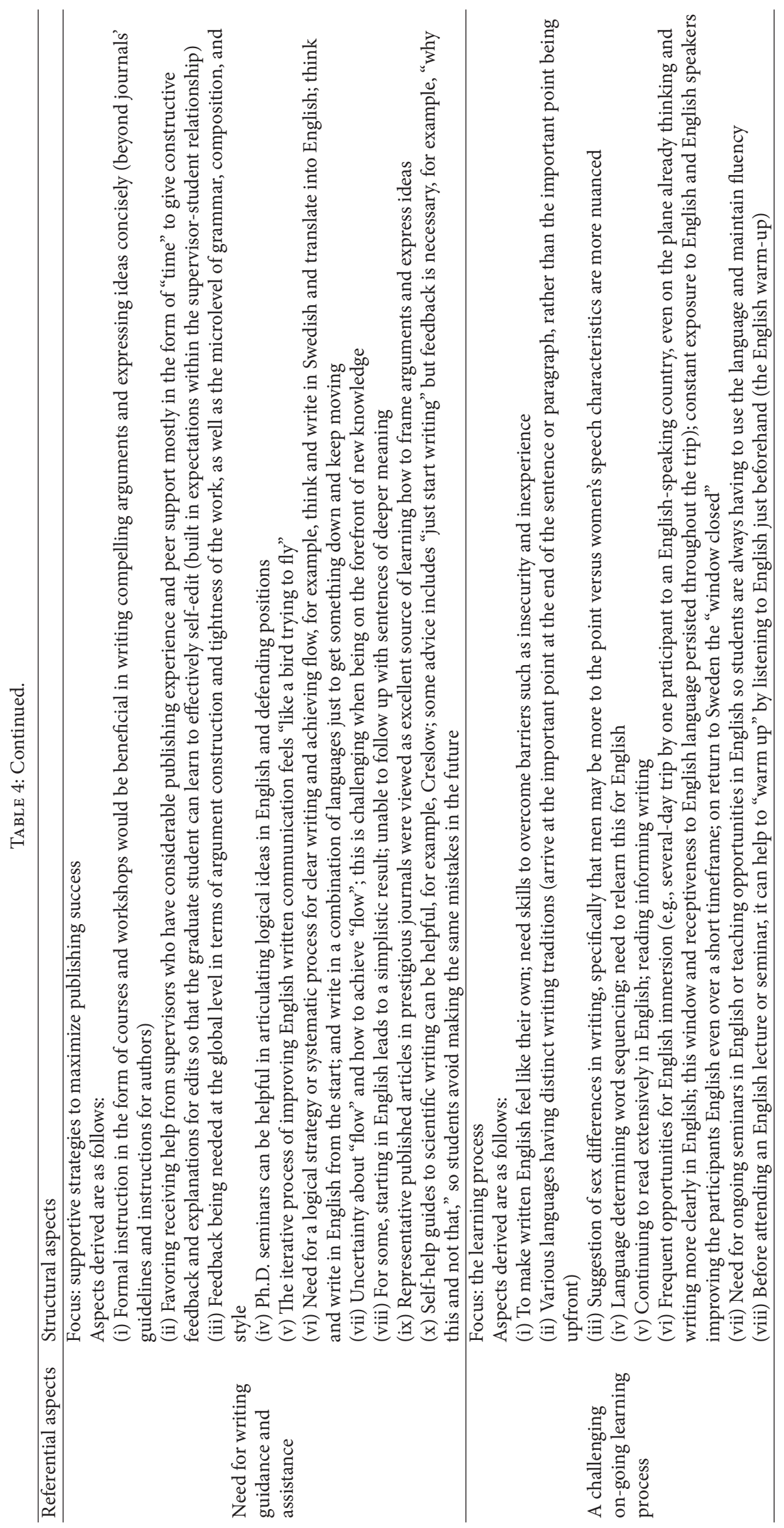


acknowledged that to "...write one sentence and to deepen that thought in the next sentence...that's the challenge..." (Stud-P9, page 8). Student 12 alluded to the experience as "wearing down one's motivation, the momentum gets lost, muted and that creates frustration" (Stud-P12, page 9).

Regarding journal styles, Student 11 stated that "when I look at the guidelines in certain journals, they are not so good. . .they are sort of vague and you have to read a couple of articles or a lot articles from that journal..." (Stud-P11, page 9).

Student 1 talked about the expectation of negative reviews related to the English "And may be you are a little bit afraid what the reviewers say about the text, may be they are hard. ..they have to say what is wrong of course, but in a way you are very much taken down" (Stud-P1, page 13).

Supervisors perceive writing as an academic responsibility (supervisor referential aspect, an academic responsibility), whereas students view the need to write in the short term (student referential aspect, a need to focus on the goal [an article]). Supervisor 2, for example, said that at a previous university there were financial rewards "... relating to the number of publications and the impact factor of (the journals) of those publications" (Sup-P2, page 5). Regarding writing as a shortterm goal, Student 5 stated that "finishing and completing (a manuscript) is my goal" (Stud-P5, page 4). Student 7 also made reference to the goal of reaching "... a wider audience or target group...if it was published in Swedish, not so many people could read about it..." (Stud-P7, page 4).

Regarding writing English being a "disadvantageous" struggle, Supervisor 5 wished that "the English-speaking world better understood that they speak and write in their native tongue... it's very much a hegemoneous (hegemonical) English world (academia), and I... wish some more humbleness from the side of the journal. .." (Sup-P5, page 15). He/she further acknowledged "how to you go about it (addressing the issue), but it is a barrier if you feel ridiculed because you write something and you make mistakes" (Sup-P5, page 16). Supervisor 2 added that "after a while if you survive this your skin gets harder" (Sup-P2, page 16), however Supervisor 5 expressed concern about the risk that "you become a perpetrator yourself" (Sup-P5, page 16).

The use of punishing tactics by reviewers, for example, ridicule/sarcasm and "making fun of your shortcomings," was viewed as counterproductive (Sup-P2 and Sup-P5, page 15). Supervisor 1 added that "I once had it (manuscript) come back a couple of times that I needed to, to get a better flow in the introduction...there was flow...I never understood the... criticism" (Sup-P1, page 16).

Supervisor 5 raised the issue of "beyond language but not less connected to writing in English is also the cultural lens" and "how you present an argument, what is flow, what is directness..." (Sup-P5, page 17). Regarding an academic cultural lens, he/she acknowledged that this can manifest in referencing, that is, "...cite too many Russian sources you would be rejected; you need 'proper' sources from the western world, those sorts of things are very hard to pinpoint..." (Sup-P5, page 17). Supervisor 2 also added that “...when we publish internationally we always have to...describe the Swedish healthcare system. ...when. ..I am the reviewer for US manuscripts they take it for granted that we understand their system..." (Sup-P2, page 18).

Improving proficiency in English scientific writing was identified as essential by both groups (supervisor referential aspect, need to master the written English language; and student referential aspect, need for guidance and assistance); however, the means of achieving this were distinct. Supervisors discussed the importance of facilitating a culture of English communication in academia. Supervisor 1 suggested that not only "using the time we have better" but also "better awareness in the organization of this need..." (Sup-P1, page 19). Overall, supervisors favored "hands on" feedback and small writing groups with a native English speaker to go over writing examples and sharing edited versions with each other. Supervisor 1 said "... not so much about content but how you formulate it, so you would sit together may be with different disciplines; it would be fascinating with an (native) English speaker, a scientist, discussing what I want to express, so you just take pieces of it and you discuss it, and you read each other's" (Sup-P1, page 19). Supervisor 2 suggested that in departmental seminars "...you sit together around a table and you work intensely with both content and structure and so on..." (Sup-P2, page 20). Supervisor 1 added that "once in a while we have a guest lecturer here and it is a researcher; it would be really nice to sit down for a short meeting and discuss. . .how should I express this with different examples" (Sup-P1, page 21). Supervisor 3 emphasized that even informal opportunities to communicate are worthwhile, specifically, "to start to talk. . .if you get to use the language in the corridors, in the 'fika' (coffee) room, I think that is important" (Sup-P3, page 22). Supervisor 5 noted “... why do not people want to teach in English?... if you teach in English it is easy to write in English, if you write in English it is more likely you can teach in English" (Sup-P5, page 22). Supervisor 1 added that "resistance about teaching in English...is not only from colleagues but actually...the students" (Sup-P1, page 23). Supervisor 5 added that "...when I started teaching... I taught only in English and that's how I started writing in English...you just learned by doing..." (Sup-P5, page 23).

Supervisors and students use various supports including handbooks. Supervisor 4 acknowledge that one handbook was particularly helpful as it describes "...how to think about language, not grammar in itself...ways to structure a text. ..words to start an argument. . .reach between two different ideas" and that it gives advice in a "friendly tone" (Sup-P4, pages 23 and 24). Student 9 referred to a particular resource: "...our favorite, he (author) wrote (recommended) to write every day...just to start writing. . . play with the words then come to the more scientific writing..." (Stud-P9, page 11).

The students focused on additional strategies. Student 6 acknowledged that "I read other articles...to find out. . .how they are using argumentation... expressing things..." (StudP6, page 10). Regarding scientific writing courses, Student 2 said that such a course "was quite helpful for you to figure out what is exactly scientific writing, what is the style; to move to introduction you have three 'moves', and then there is the issue of grammar. . .but you have to learn about the style and the flow...so you can construct your paper...scientifically" (Stud-P2, page 10). 
Students agreed with Student 12 who emphasized the role of "a good supervisor and good friends that love to read English scientific text" (Stud-P12, page 10) as an important means of receiving concrete feedback and improving a manuscript. Student 7 acknowledged the importance of specific feedback: “...the details of writing, not only (that) this is wrong...I need to know why it is wrong...what I should write instead." Student 7 described a shared concern about avoiding similar mistakes in the future: "I have to improve my English... not (necessarily) for specific text but next time when I write something similar...I do not do the same mistake..." (Stud-P7, page 11).

4.2. Synthesis. Nonnative English-speaking doctoral supervisors and students in Sweden have unique lived experiences with scientific writing, with similarities and distinctions. Thus, they may benefit from specifically tailored writing supports vis-à-vis maximizing the rewards of the writing experience, reducing challenges, increasing facilitators, and reducing barriers $[17,18]$. Supervisors urged editors and reviewers to provide more constructive rather than destructive feedback about language and expression. Opportunity to communicate in English was described as a means of keeping English language use current rather than restricting use of English to manuscript writing. Instructional objectives, formats, and feedback mechanisms in courses on scientific writing in English are indicated that extend beyond the standard journal advice and instructions for authors.

Although replication/extension studies are needed, steps need to be taken to ensure that the work of nonnative English-speaking scholars and trainees is effectively and fairly critiqued and evaluated by native English-speaking reviewers [19]. Standards must be preserved; however, reviewers need to be educated about the challenges of nonnative English speakers so their efforts can be maximally supported, to ensure their voices and perspectives are proportionately reflected in the literature [11]. Given that scientific writing in English is laborious for nonnative English speakers and precludes their publishing at rates comparable to their native English-speaking peers, such efforts could enable nonnative English-speaking scholars and trainees to maximize their scholarly productivity and contribution to the generation of new knowledge and science overall. Publishing an article in the language of an author's choice is not likely to be an option. However, reprinting articles in two or more languages is conceivable and desirable. Education needs to be provided for reviewers and editors to increase their awareness of the challenges of nonnative English contributors and maximize ways of supporting their efforts to publish. For example, this could include guidelines for providing constructive feedback that maximizes the contribution of the content and focus of a manuscript by improving the quality of English expression. Enabling the efforts of reviewers and editors in this way may curb the growth industries of ghost writing and predatory journals and potential for duplicate publication and plagiarism [20-22].

4.3. Conclusion. The efforts of nonnative English-speaking scholars and trainees could be more effectively supported in the peer-review process in academia, a process that is dominated by native English-speaking editors and reviewers. Targeted resources are needed for these contributors, and for editors and reviewers regarding their providing constructive and enabling feedback. Such supports would not only facilitate publication being a more rewarding and constructive experience for both parties, but also help limit systematic bias in the review process. In turn, this would help ensure that the voices of nonnative English-speaking contributors are more proportionally reflected in the scientific literature.

\section{Conflict of Interests}

The authors declare that there is no conflict of interests regarding the publication of this paper.

\section{References}

[1] T. H. Baron, "ABC's of writing medical papers in English," Korean Journal of Radiology, vol. 13, supplement 1, pp. S1-S11, 2012.

[2] S.-T. Hong, "Launching a new section for the Journal of Korean Medical Science: focusing on editing, writing, and publishing issues," Journal of Korean Medical Science, vol. 29, no. 1, p. 1, 2014.

[3] P. A. Hall, "Getting your paper published: an editor's perspective," Annals of Saudi Medicine, vol. 31, no. 1, pp. 72-76, 2011.

[4] C. T. Quinn and A. J. Rush, "Writing and publishing your research findings," Journal of Investigative Medicine, vol. 57, no. 5, pp. 634-639, 2009.

[5] W. Zhang, “Ten simple rules for writing research papers," PLoS Computational Biology, vol. 10, no. 1, Article ID e1003453, 2014.

[6] G. M. Liumbruno, C. Velati, P. Pasqualetti, and M. Franchini, "How to write a scientific manuscript for publication," Blood Transfusion, vol. 11, no. 2, pp. 217-226, 2013.

[7] P. R. Shankar, "What do reviewers look for in an original research article?" Journal of the Nepal Medical Association, vol. 52, no. 186, pp. 95-101, 2012.

[8] J. Smyth, J. Verweij, M. D'Incalci, and L. Balakrishnan, “"The Art Successful Publication' ECCO 13 workshop report," European Journal of Cancer, vol. 42, no. 4, pp. 434-436, 2006.

[9] B. L. La Madeleine, "Lost in translation," Nature, vol. 445, no. 7126, pp. 454-455, 2007.

[10] F. Gannon, "Language barriers," EMBO Reports, vol. 9, no. 3, p. 207, 2008.

[11] A. S. Canagarajah, A Geopolitics of Scientific Writing, University of Pittsburg Press, Pittsburgh, Pa, USA, 2002.

[12] S. M. R. Vasconcelos, M. M. Sorenson, J. Leta, M. C. Sant'Ana, and P. D. Batista, "Researchers' writing competence: a bottleneck in the publication of Latin-American science?" EMBO Reports, vol. 9, no. 8, pp. 700-702, 2008.

[13] M. A. Marlow, "Writing scientific articles like a native English speaker: top ten tips for portuguese speakers," Clinics, vol. 69, no. 3, pp. 153-157, 2014.

[14] F. Marton, "Phenomenography-describing conceptions of the world around us," Instructional Science, vol. 10, no. 2, pp. 177200, 1981.

[15] C. Yates, H. L. Partridge, and C. S. Bruce, "Exploring information experiences through phenomenography," Library and Information Research, vol. 36, no. 112, pp. 96-119, 2012. 
[16] B. Reed, Phenomenography As a Way to Research the Understanding by Students of Technical Concepts, Núcleo de Pesquisa em Tecnologia da Arquitetura e Urbanismo (NUTAU): Technological Innovation and Sustainability, São Paulo, Brazil, 2006.

[17] H. Glasman-Deal, Science Research Writing for Non-Native Speakers of English, Imperial College Press, London, UK, 2010.

[18] R. A. Day and N. Sakaduski, Scientific English. A Guide for Scientists and Other Professionals, Greenwood, Santa Barbara, Calif, USA, 3rd edition, 2011.

[19] R. Meneghini and A. L. Packer, "Is there science beyond English? Initiatives to increase the quality and visibility of non-English publications might help to break down language barriers in scientific communication," EMBO Reports, vol. 8, no. 2, pp. 112-116, 2007.

[20] M.-C. Roland, "Publish and perish. Hedging and fraud in scientific discourse," EMBO Reports, vol. 8, no. 5, pp. 424-428, 2007.

[21] I. Masic, "Plagiarism in scientific research and publications and how to prevent it," Materia Socio Medica, vol. 26, no. 2, pp. 141146, 2014.

[22] L. Logdberg, "Being the ghost in the machine: a medical ghostwriter's personal view," PLoS Medicine, vol. 8, no. 8, Article ID e1001071, 2011. 

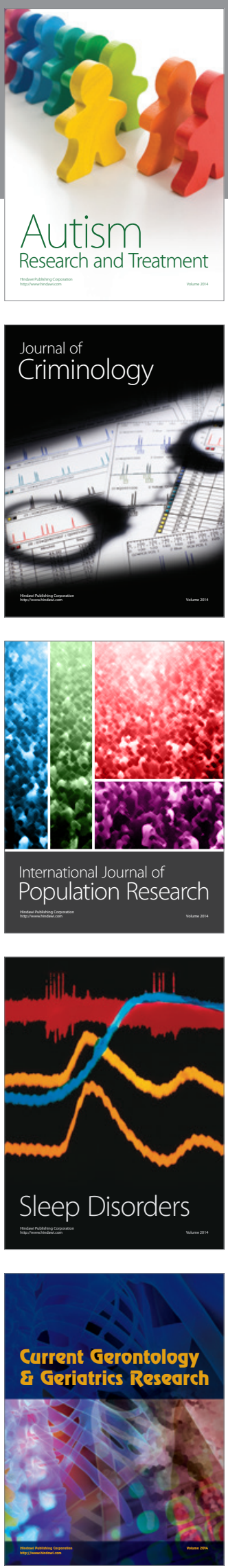
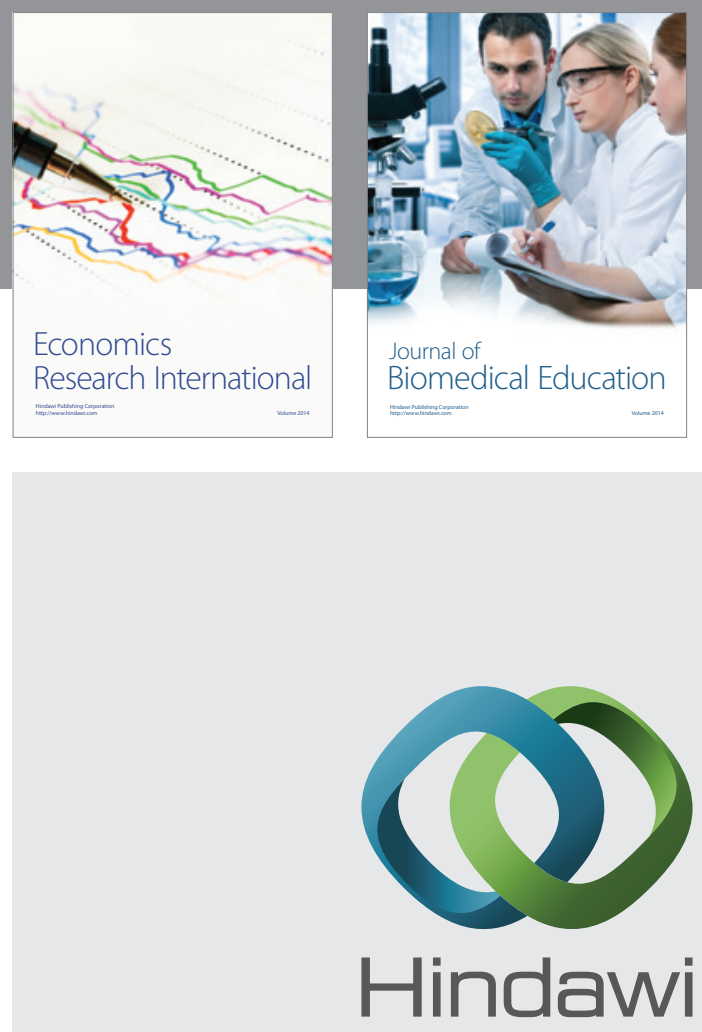

Submit your manuscripts at

http://www.hindawi.com
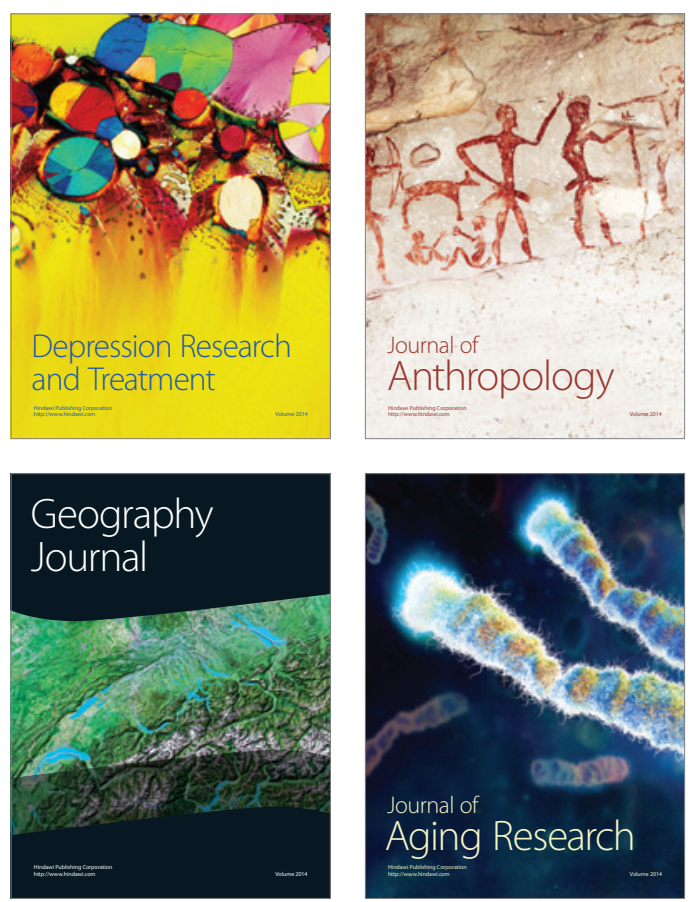
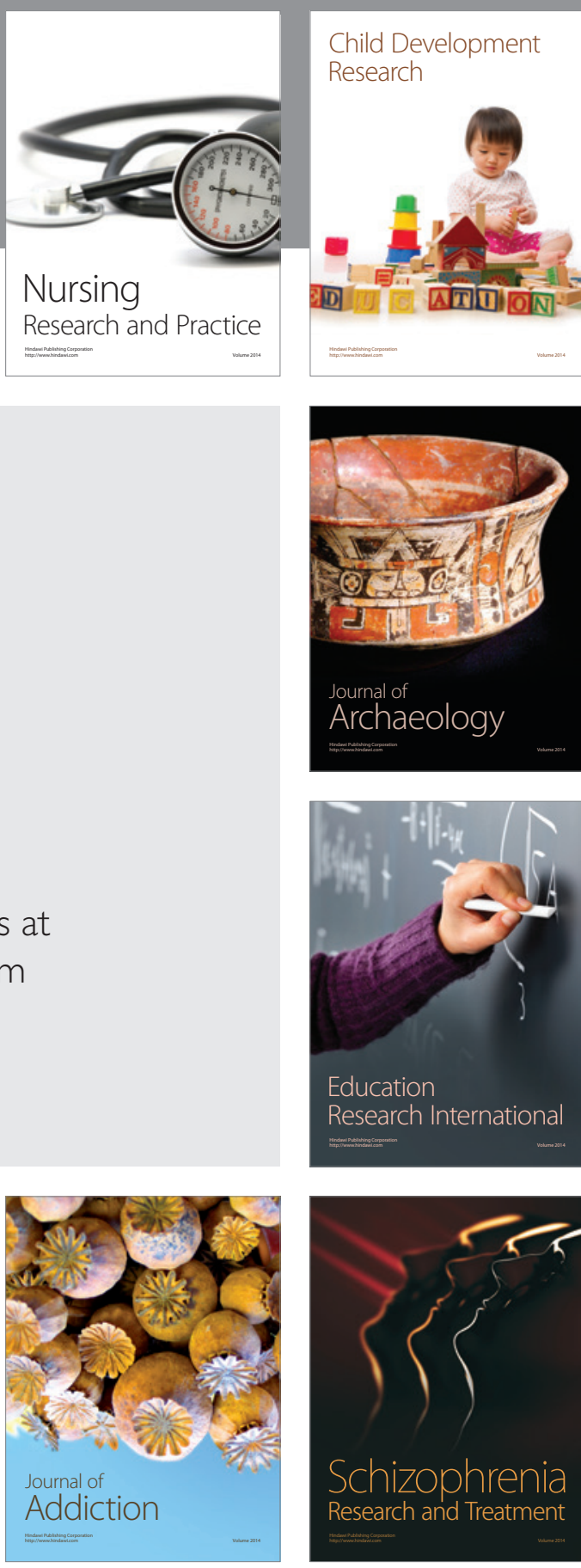

(D)
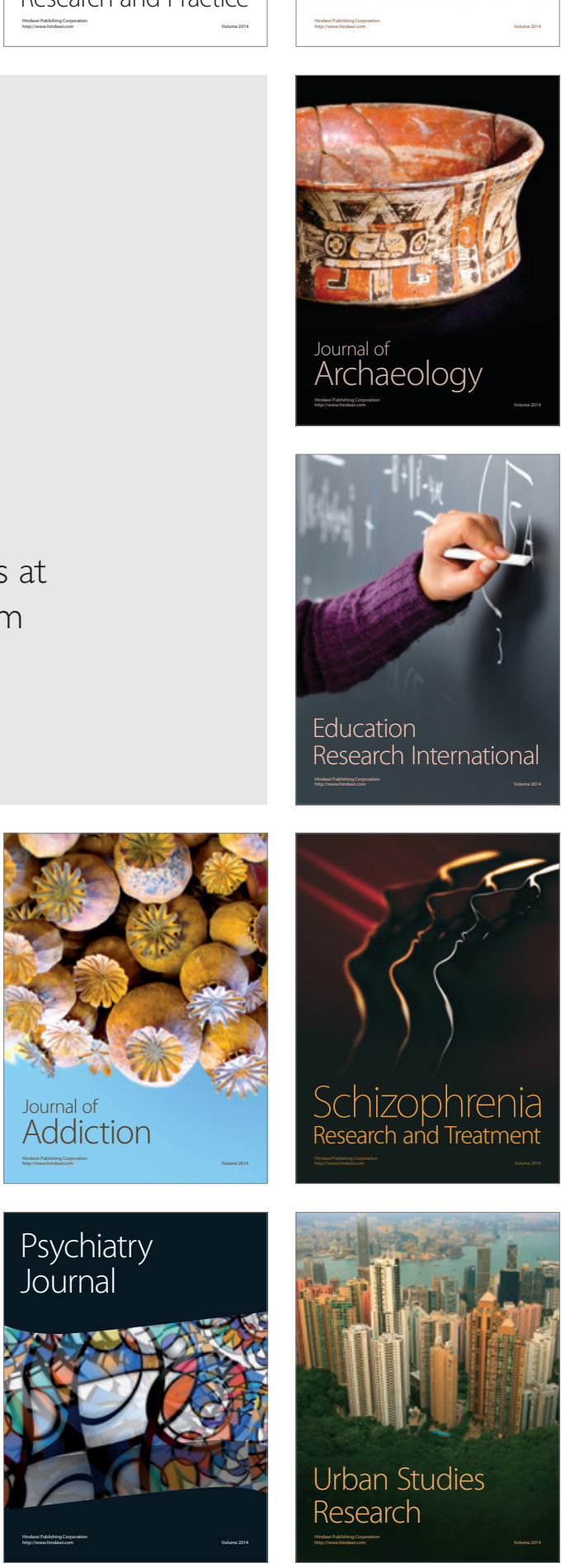